\title{
Interaction between the mouse homologue of CD99 and its ligand PILR as a mechanism of T cell receptor-independent thymocyte apoptosis
}

\author{
Hyo Jin Park ${ }^{1 *}$, Young Larn Ban ${ }^{2 *}$, Dahye Byun ${ }^{3}$, \\ Seong Hoe Park ${ }^{1,2}$ and Kyeong Cheon Jung ${ }^{1,2,4}$

\section{${ }^{1}$ Department of Pathology} \\ ${ }^{2}$ Department of Immunology \\ ${ }^{3}$ Department of Biomedical Sciences \\ Seoul National University College of Medicine \\ Seoul 110-799, Korea \\ ${ }^{4}$ Corresponding author: Tel, 82-2-740-8265; \\ Fax, 82-2-763-6625; E-mail, jungkc66@snu.ac.kr \\ *These authors contributed equally to this work. \\ DOI 10.3858/emm.2010.42.5.037
}

Accepted 2 March 2010

Available Online 8 March 2010

Abbreviations: DN, double negative; DP, double-positive; FTOC, fetal thymic organ culture; PI, propidium iodide; PILR, paired immunoglobuling-like type 2 receptor; SEB, staphylococcal enterotoxin B; SP, single-positive; $\Delta \psi \mathrm{m}$, mitochondrial membrane potential

\begin{abstract}
Here, we show that the interaction between two membrane proteins, the mouse homologue of CD99 (designated D4) and its ligand, paired immunoglobulin-like type 2 receptor (PILR), is one of the major mechanisms of thymocyte apoptosis. Using the polymeric fusion protein of PILR and IgG1 (PILR-Ig), we demonstrated that $\mathrm{D} 4$ ligation in the absence of $T$ cell receptor (TCR) engagement leads to the induction of apoptosis, mainly at the double-positive stage of thymocytes. This was further confirmed by a blocking study in which blocking the interaction between D4 and PILR by soluble D4 protein led to reduced apoptosis in the fetal thymic organ culture with wild type and TCR $\alpha^{-t}$ mice. Furthermore, the dissection of intracellular signaling pathway demonstrated that $\mathrm{D} 4$ cross-linking led to caspase activation without any change in mitochondrial membrane potential. Based on these data, we propose a mechanism for thymocyte depletion in which the interaction between D4 and PILR delivers an active signal.
\end{abstract}

Keywords: apoptosis; CD99 antigen, mouse; PILR protein, mouse; $\mathrm{T}$ cell receptor; thymocyte

\section{Introduction}

In the thymus, most thymocytes that have not successfully re-arranged their TCR genes or that express a receptor with subthreshold avidity for self-antigen/MHC enter a default apoptosis pathway (Chan et al., 1993; Crump et al., 1993; Cresswell, 1998). This process occurs among the immature $\mathrm{CD} 4^{+} \mathrm{CD} 8^{+}$double positive (DP) thymocytes and involves mechanisms that remain elusive (Cohen, 1991). In an effort to understand apoptosis during T-cell development, significant attention has been focused on the possibility of interactions between membrane proteins generating an active death signal, as opposed to the widely accepted simple default pathway (Lesage et al., 1997; Grebe et al., 2004; Jung et al., 2004). The ligation of molecules, i.e., CD8 (Grebe et al., 2004), CD24 (Jung et al., 2004), and CD45 (Lesage et al., 1997), can induce thymocyte death in the absence of TCR engagement. However, a major drawback to these studies is that they used a monoclonal antibody as the ligand, which creates a condition that differs from the normal physiological environment for thymocyte development.

The 32-kDa surface glycoprotein CD99 is highly expressed on cortical thymocytes, whereas it is moderately expressed on more differentiated cell types (Ellis et al., 1994). CD99 has been implicated in a number of cell-cell adhesion and cell activation phenomena. In addition, CD99 ligation leads to the inhibition of leukocyte diapedesis (Schenkel et al., 2002), as well as the induction of homotypic aggregation (Bernard et al., 1995) and apoptosis of DP thymocytes (Bernard et al., 1997). Moreover, CD99 engagement on immature thymocytes induces the up-regulation of MHC and TCR molecules (Choi et al., 1998). A novel mouse homologue of human CD99, D4, has been identified as a ligand of the paired Ig-like type 2 receptor (PILR) (Shiratori et al., 2004). We previously suggested that D4 is homologous to CD99 based on high amino acid sequence homology, similar patterns of distribution in various tissues, and shared functional characteristics with several highly conserved amino acid motifs (Park et al., 2005). 
A

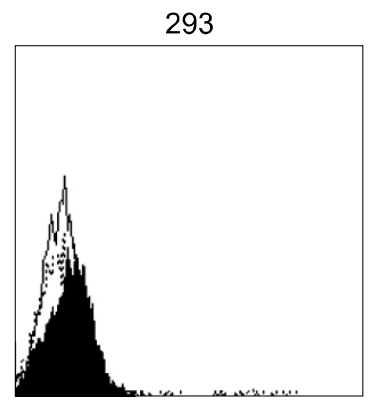

C

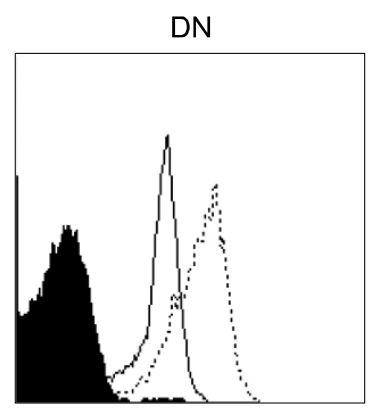

D

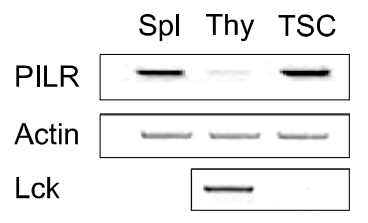

B

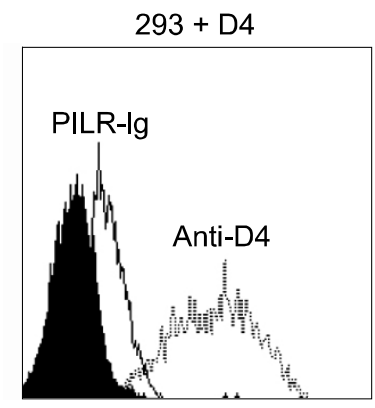

IP with: PILR-Ig

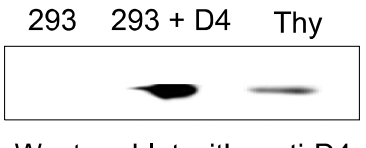

Western blot with: anti-D4
DP

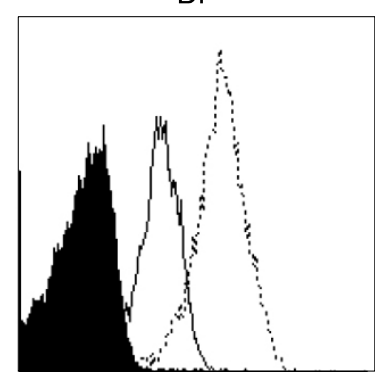

CD4SP

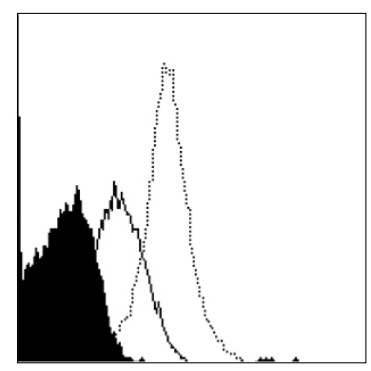

Control

PILR-Ig

........ Anti-D4

Figure 1. PILR-Ig recognizes D4. (A) The 293 cells were transfected with the D4 expression vector (293 + D4). The parental 293 cells and the D4 transfectants were treated with either PILR-Ig (solid line) or control-lg (filled histogram), followed by staining with PE-conjugated goat-anti-human IgG or PE-conjugated anti-D4 monoclonal antibody (dashed line). (B) Cell lysates of parental 293 cells (293), D4-transfected 293 cells $(293+$ D4) and thymocytes (Thy) were immunoprecipitated with PILR-lg. The immunoprecipitates were immunoblotted with anti-D4 mAb. (C) DN, DP, and CD4 ${ }^{+}$SP thymocytes were isolated by magnetic sorting and the expression of D4 in each subset was examined using PILR-lg (solid line), control-Ig (filled histogram), and anti-D4 monoclonal antibody (dashed line). (D) Transcripts of PILR in thymocytes (Thy) and thymic stromal cells (TSC) was analyzed by RT-PCR. As a positive control, splenocytes (Spl), which are also known to express PILR, were included. Transcripts of $\beta$-actin were used as a standard. Data are representative of two or three similar experiments.

On the basis of these findings, we hypothesized that D4 plays a certain functional role during thymocyte development. To elucidate the role of D4 in the process of T-cell development, we investigated whether D4 ligation by PILR-Ig induces any physiological response in murine thymocytes.

Here, we show that thymocytes undergo cell death after D4 cross-linking with PILR-Ig in the absence of TCR/CD3 engagement. Furthermore, the blockage of D4 signals using a soluble D4 (fusion protein of D4 and Fc portion of human IgG1, D4-Fc) in fetal thymic organ culture (FTOC) resulted in increased cortical cellularity. Finally, we demonstrated that apoptosis following D4 cross-linking leads to caspase activation without any change in mitochondrial membrane potential $(\Delta \psi \mathrm{m})$. These results suggested that $D 4$ engagement with its ligand plays some role in thymic education process via affecting the thymocyte apoptosis.

\section{Results}

\section{PILR is a ligand for D4}

To confirm that PILR is a ligand for D4, 293 cells transfected with the D4 gene were stained with PILR-Ig protein and PE-conjugated anti-human immunoglobulin. Subsequent flow cytometric analysis confirmed the interaction between PILR and D4 (Figure 1A). In addition, when D4 was precipitated with PILR-Ig from cell lysates of D4 transfectants and thymocytes, D4 was clearly detected as a $17-\mathrm{kDa}$ protein, which was not identified in untransfected 293 cells (Figure 1B), 
A

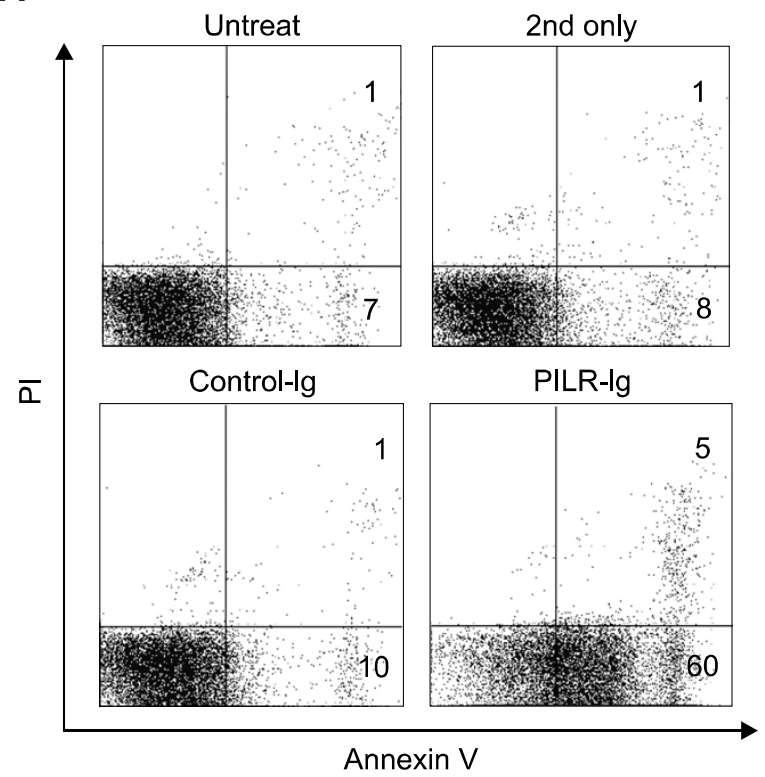

C

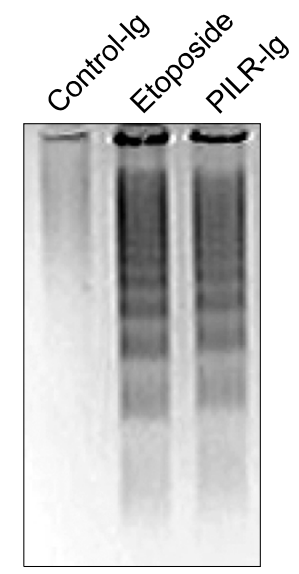

B

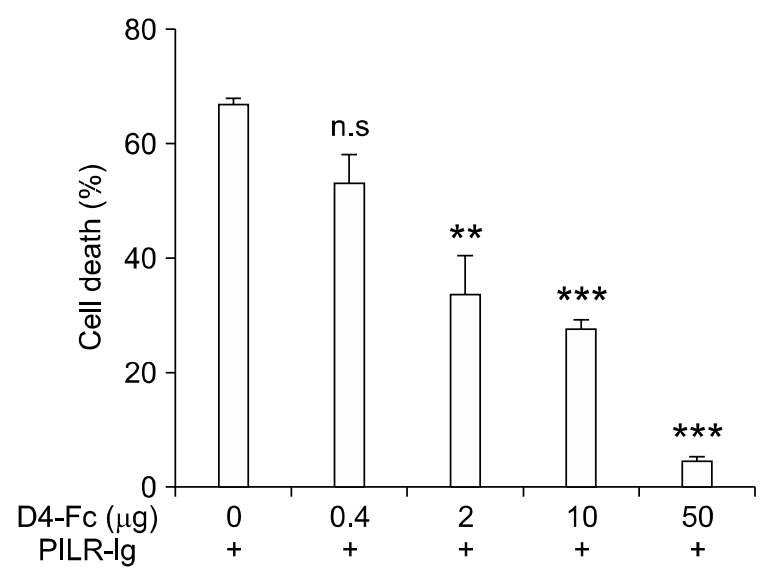

D

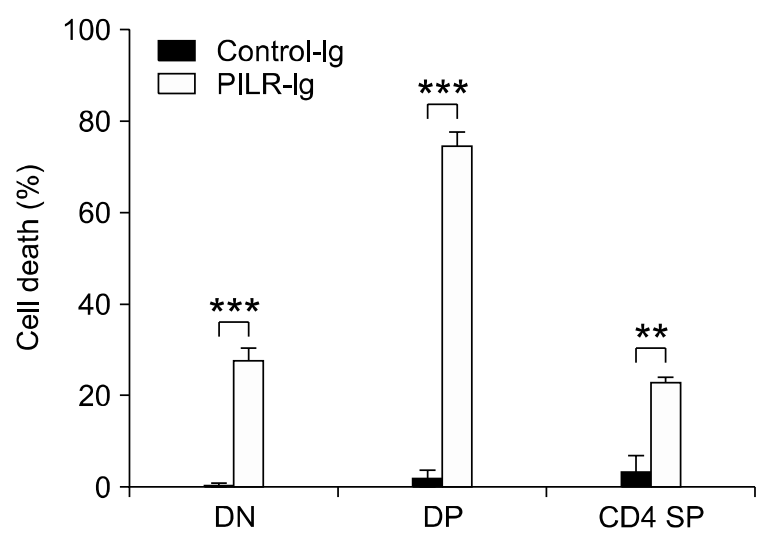

Figure 2. Cross-linking of D4 by PILR-lg induces apoptosis in murine thymocytes. (A) Thymocytes were cultured for $6 \mathrm{~h}$ at $37^{\circ} \mathrm{C}$ in the presence of PILR-lg $(20 \mu \mathrm{g} / \mathrm{ml})$ or control-lg $(20 \mu \mathrm{g} / \mathrm{ml})$ with secondary antibody $(20 \mu \mathrm{g} / \mathrm{ml})$. Apoptosis was evaluated by annexin V and PI staining. One representative flow cytometry result of five independent experiments is shown. (B) PILR-Ig was pre-incubated for $1 \mathrm{~h}$ with various dosages of D4-Fc (0-50 $\mu \mathrm{g} / \mathrm{ml})$ at $4^{\circ} \mathrm{C}$, then added to thymocytes cultures and incubated for $6 \mathrm{~h}$ at $37^{\circ} \mathrm{C}$ in the presence of this complex with secondary Ab. The percentages of dead cells from each sample were quantitated and normalized as described in Methods. Results are expressed as the mean \pm SEM of triplicate samples and representative of two independent experiments. n.s. not significant, ${ }^{* *} P<0.01$, ${ }^{* * *} P<0.001$ comparing D4-Fc treated versus untreated thymocytes. (C) Thymocytes were treated in the same manner as in (A), and the extracted DNA from each sample was separated by $3 \%$ agarose gel electrophoresis. Etoposide, which is known to induce the typical DNA ladder formation, was used as a positive control. Data are representative of two independent experiments (D) The sorted DN, DP, and CD4 ${ }^{+}$SP thymocytes were incubated with PILR-Ig or control-lg in the presence of secondary Ab. The percentage cell death was measured and normalized. Results are expressed as the mean \pm SEM of triplicate samples and representative of two independent experiments. ${ }^{* *} P<0.01,{ }^{* * *} P<0.001$.

confirming that PILR-Ig recognizes D4 (Shiratori et al., 2004).

D4 expression was detected at all stages of thymocyte development. However, the expression of D4 varied depending on the specific developmental stage, with expression being progressively down-regulated from double negative (DN) to mature single positive (SP) thymocytes (Figure 1C).

We also compared the expression profile of PILR between thymocytes and thymic stromal cells, to identify the major cellular source of PILR. PILR transcripts were detected predominantly in thymic stromal cells, whereas the transcripts were 
A

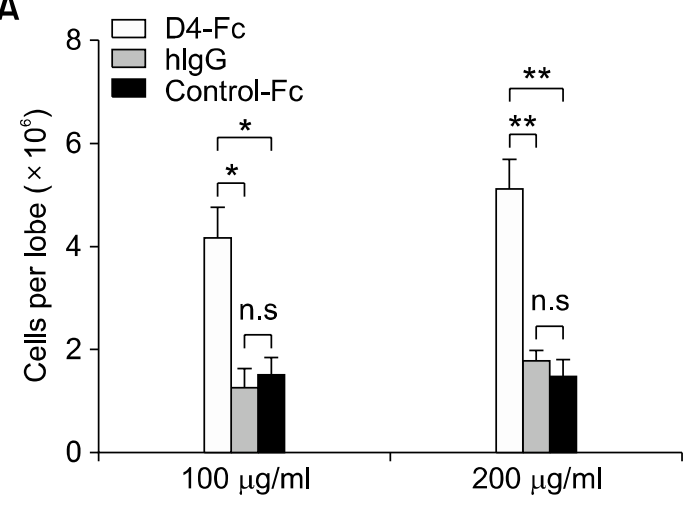

C

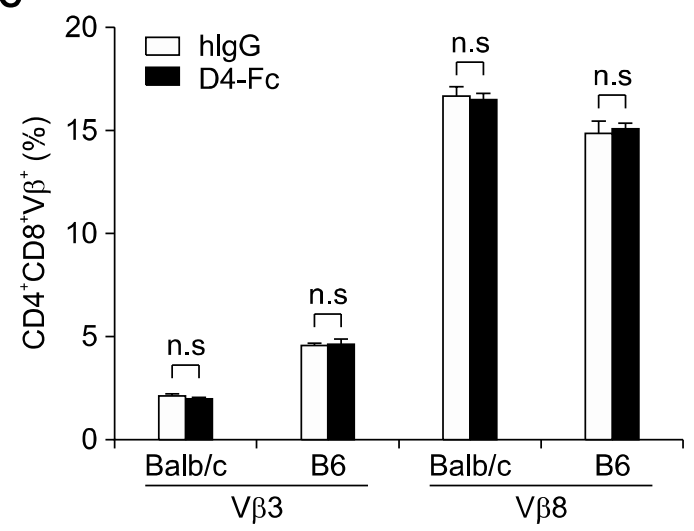

E

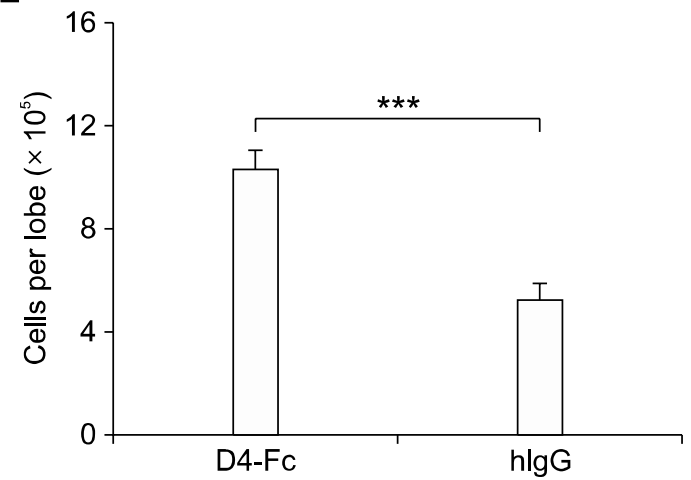

B

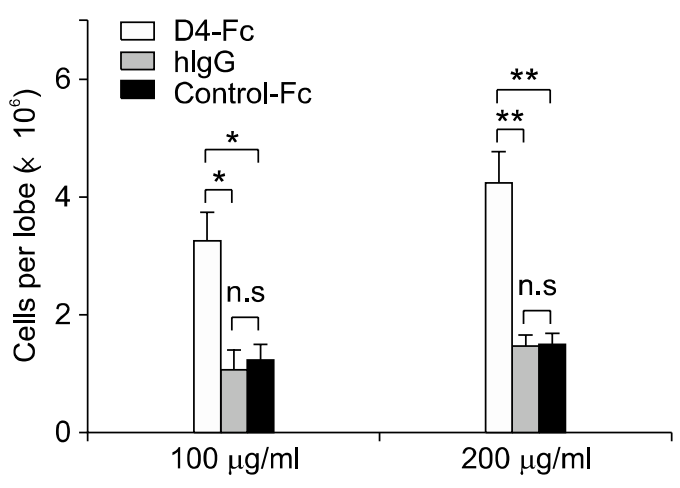

D

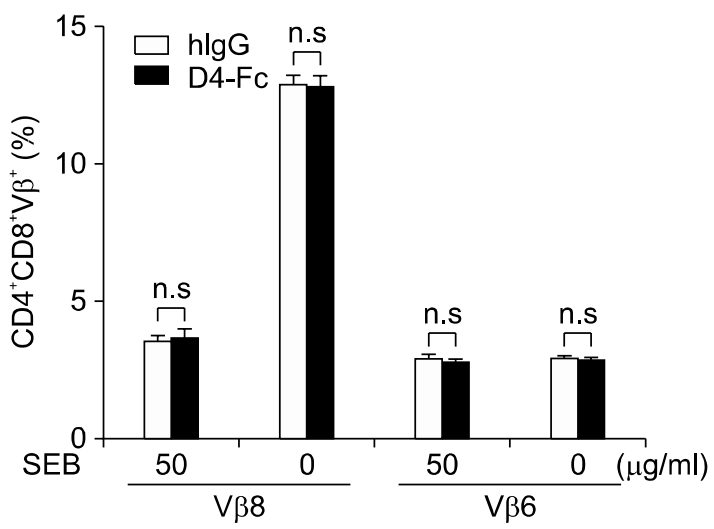

$\mathbf{F}$

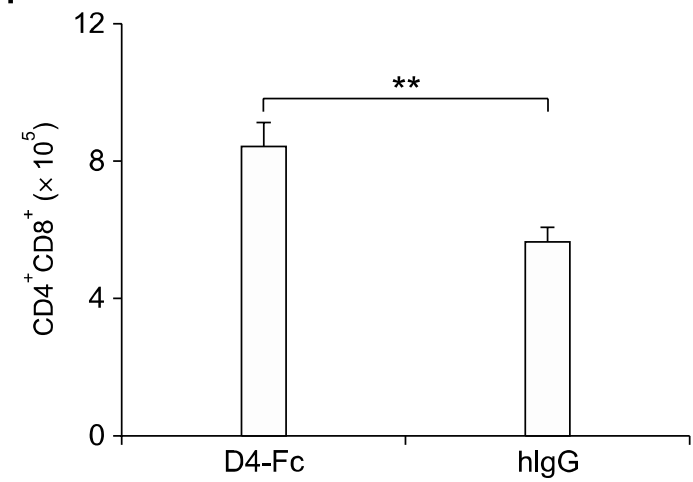

Figure 3. The blockage of D4 signaling via D4-Fc leads to an increase in the number of DP thymocytes in FTOC from wild-type and TCR $\alpha^{-/}$mice, but does not influence superantigen-induced deletion. (A and B) Fetal Day 15.5 thymi from C57BL/6 mice were separated into lobes and cultured for 5 days in medium with $100 \mu \mathrm{g} / \mathrm{ml}$ or $200 \mu \mathrm{g} / \mathrm{ml}$ of D4-Fc (white bars), human lgG (shaded bars), or control CD86-Fc (black bars). Thymocytes were isolated, counted, and stained with fluorochrome-conjugated antibodies to CD4 and CD8. The absolute numbers of total (A) and DP (B) thymocytes were calculated using the data from the flow cytometric analysis. Results are expressed as the mean \pm SEM of triplicate samples and representative of two independent experiments. (C and D) Fetal Day 15.5 thymic lobes from Balbc/c and C57BL/6 (B6) mice were cultured for 5 days (C) or for 7 days (D) in medium with control human lgG (white bars) or D4-Fc (black bars), and SEB $(50 \mu \mathrm{g} / \mathrm{ml}$ ) was added for the final $40 \mathrm{~h}$ of the culture period (D). Thymocytes were isolated and stained with fluorochrome-conjugated antibodies to CD4, CD8, TCRV $\beta 3$ (Mtv-9-reactive), TCRV $\beta 8$ (SEB-reactive), and TCRV $\beta 6$ (non-reactive to superantigen). The percentages of each TCR V $\beta^{+} D P$ thymocytes were calculated using the data from the flow cytometric analysis. Results are expressed as the mean \pm SEM of tridecaplicate $(n=13 ; C)$ or nonaplicate $(n=9 ; \mathrm{D})$ samples and representative of two similar experiments. (E-F) Fetal Day 15.5 thymic lobes from TCR $\alpha^{-1-}$ mice were cultured for 5 days in medium with control human $\operatorname{lgG}$ or D4-Fc. Thymocytes were isolated, counted, and stained with fluorochrome-conjugated antibodies to $\mathrm{CD} 4, \mathrm{CD}$, and CD3. The absolute numbers of total (E) and DP (F) thymocytes were calculated using the data from the flow cytometric analysis. Results are expressed as the mean \pm SEM of octaplicate $(n=8)$ samples and representative of two independent experiments. n.s. not significant, ${ }^{*} P<0.05,{ }^{* *} P<0.01,{ }^{* * *} P<0.001$. 
present at almost negligible levels in thymocytes (Figure 1D). Simultaneously, we measured the expression of Lck to confirm that the thymic stromal cell populations were not contaminated with thymocytes. Therefore, the PILR expression pattern supported our idea that PILR expressed on thymic stromal cells recognizes D4 on thymocytes.

\section{Cross-linking of D4 with PILR-Ig induces thymocyte apoptosis}

To examine the functional role of D4, we investigated the effect of the D4-PILR interaction on the development of mouse thymocytes. When mouse thymocytes were treated with PILR-Ig in the presence of secondary antibody for $6 \mathrm{~h}$, more than $50 \%$ of the thymocytes underwent apoptosis (Figure 2A). In contrast, treatment with the control chimeric Ig molecule (CD86-lg) resulted in a rate of apoptosis that was comparable to that of the Ig-untreated culture. The PILR-induced apoptosis was profoundly inhibited by treatment with D4-Fc (Figure 2B), which indicates that PILR induced thymocyte apoptosis via D4 engagement. Moreover, internucleosomal DNA cleavage, which is a hallmark of either caspase-3 or caspase- 7 activity (Hirata et al., 1998), was clearly observed in the form of DNA laddering (Figure $2 \mathrm{C}$ ), which strongly suggests that the apoptosis induced by D4 engagement involved caspase activation.

Because the expression level of D4 varied according to the developmental stage of the thymocytes (Figure 1C), we tested the sensitivity of individual developmental stages of the thymocytes to D4-mediated death. When thymocytes were sorted into DN, DP, and SP fractions and cultured in the presence of PILR-Ig and the cross-linking antibody, the DP thymocytes were much more sensitive to D4 cross-linking than were the DN and SP thymocytes (Figure 2D). These data suggest that thymocyte death via D4 predominantly affects DP cells and occurs in a TCR-independent manner.

\section{Blocking the interaction between D4 and PILR could rescues cortical thymocytes that could not positively selected}

To evaluate these findings in more detail, experiments were conducted in the FTOC system, as this system more closely resembles the in vivo system. In control groups in which cultures of fetal thymic organs from normal C57BL/6 mice were treated with control Fc-containing proteins (i.e., normal human IgG1or human CD4-Fc fusion protein), thymic cellularity was not affected (Figures $3 \mathrm{~A}$ and $3 \mathrm{~B}$ ). However, treatment with the D4-Fc dimer in the parallel cultures increased the numbers of thymocytes in a dose-dependent manner (Figures $3 \mathrm{~A}$ and $3 \mathrm{~B}$ ), suggesting that the D4-Fc dimer might prolong the survival of developing thymocytes by neutralizing endogenous PILR on thymic stromal cells.

During thymic education, several mechanisms are involved in thymocyte apoptosis, one of which is negative selection. Thus, we investigated whether the increased cellularity of $\mathrm{CD} 4^{+} \mathrm{CD} 8^{+}$thymocytes might be caused by rescue from impaired negative selection, using two model systems of negative selection of thymocytes: one mediated by an endogenous superantigen and the other by an exogenously administered superantigen in BALB/C mice. In BALB/C FTOC, the population of TCRV $\beta 3^{+}$ cells are reduced because of negative selection by the Mtv-6-encoded endogenous superantigen in compared to the C57BL/6 FTOC (Frankel et al., 1991). Based on this, when BALB/C FTOC was treated with D4-Fc, the percentage of the TCRV $\beta 3^{+}$ population was not increased, demonstrating that blocking the interaction between D4 and PILR by $\mathrm{D} 4-\mathrm{Fc}$ treatment did not affect the negative selection of the Mtv-6-reactive TCRV $\beta 3^{+}$DP cells (Figure $3 \mathrm{C}$ ). This was also the case when exogenous superantigen was used. The addition of staphylococcal enterotoxin B (SEB) to BALB/C FTOC's deleted most of the developing TCRV $\beta 8^{+}$ thymocytes, but not the TCRV $\beta 6^{+}$thymocytes (Figure 3D) (Jenkinson et al., 1990). D4-Fc-treated thymic lobes remained susceptible to the deletion of TCRV $\beta 8^{+}$thymocytes by SEB (Figure 3D), which demonstrates that the blocking of D4 signaling by D4-Fc did not affect SEB-induced cortical negative selection.

In TCR ${ }^{-1-}$ mice, DP thymocytes cannot undergo positive selection because of the absence of mature TCR, and thus have an increased risk of apoptosis via death by neglect (Matsuki et al., 2002). To address whether the molecular interaction between D4 and PILR could affect this process, we performed FTOC with D4-Fc-treated $\mathrm{TCR}^{-/-}$thymic lobes and found an about twofold increase in total and DP cell numbers in the thymi cultured in the presence of D4-Fc as compared to those treated with the isotype control (human IgG1; Figures $3 E$ and $3 F$ ). Therefore, it seems that blocking the interaction between D4 and PILR could rescue the thymocytes that fail to receive survival signal via positive selection.

\section{Engagement of D4 induces caspase activation}

DNA fragmentation and the ordered disintegration of cellular organelles are established hallmarks of cells that are undergoing apoptosis, particularly in 
A

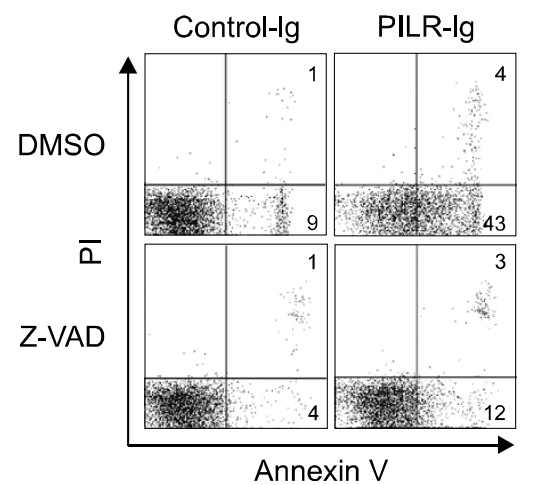

B

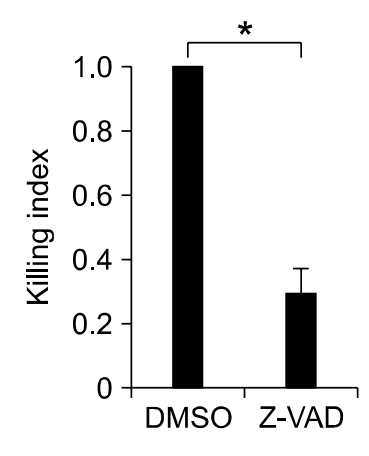

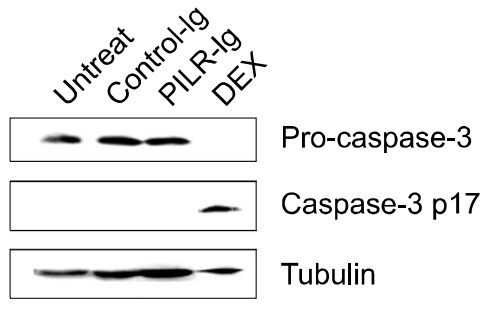

C

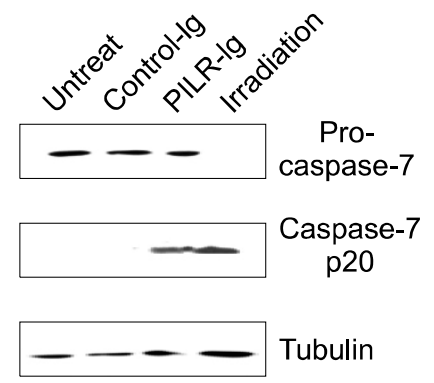

D

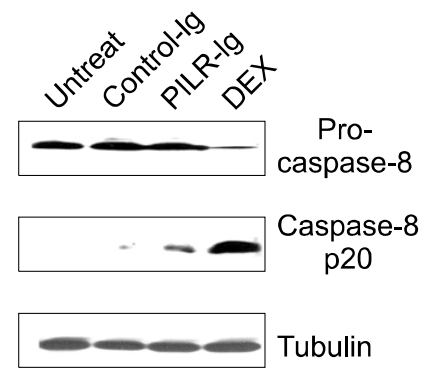

$E$

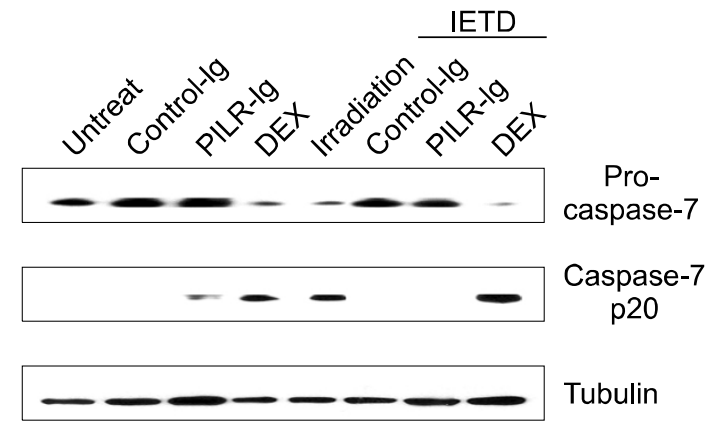

Figure 4. PILR-Ig-mediated apoptosis requires caspases. (A) Thymocytes were pretreated for $1 \mathrm{~h}$ with pan-caspase inhibitor, Z-VAD and then treated with either PILR-Ig or its isotype control in the presence of secondary antibody for $6 \mathrm{~h}$. Apoptosis was evaluated by annexin V and PI staining and one representative flow cytometry is shown. Data are pooled from three independent experiments and the normalized value for apoptosis referred to as the killing index (see Methods) is summarized in bar graph as the mean \pm SEM of triplicate samples. (B-D) Thymocytes were cultured with PILR-Ig or its isotype control, followed by treatment with secondary antibody for $6 \mathrm{~h}$ and lysis in digitonin lysis buffer. Cell lysates were separated by SDS-PAGE and immunoblotted with anti-caspase-3 antibody (B), anti-caspase-7 antibody (C), and anti-caspase-8 antibody (D). Thymocytes cultured for $6 \mathrm{~h}$ following $\gamma$ irradiation of $5 \mathrm{~Gy}$ or in the presence of $1 \mu \mathrm{M}$ dexamethasone (DEX) were used as positive controls for each caspase antibody. (E) Thymocytes were stimulated with PILR-Ig or control-Ig for $6 \mathrm{~h}$ in the absence or presence of IETD and immunoblotted with anti-caspase-7 Ab. Data were representative of two or three similar experiments.

the case of apoptosis associated with the recruitment of caspase-8 to death receptors (e.g., Fasand TNFR-mediated apoptosis) (Ashkenazi and Dixit, 1998; Rathmell and Thompson, 1999; Wallach et al., 1999). Furthermore, thymocytes that lack a productively rearranged TCR and are thus unable to recognize $\mathrm{MHC} /$ peptide complexes are deleted from the T-cell repertoire by apoptosis. Several reports have suggested that this type of death is induced by glucocorticoid receptor-mediated and caspase-dependent signal transduction (Marchetti et al., 2003; Minter and Osborne, 2003).

After confirming that signaling through D4 induces apoptosis in developing thymocytes, we further dissected D4-mediated caspase activation in immature thymocytes. There are two caspase activation pathways: (1) a mitochondrion-independent pathway in which the activation of caspase-8 followed by caspase-3 or caspase-7 predominates; and (2) a mitochondrion-dependent pathway that involves the sequential activation of caspase- 9 and caspase-3 (Li et al., 1998; Luo et al., 1998; Krammer, 2000). To elucidate the caspases responsible for D4-mediated apoptosis, the rate of apoptosis was determined with annexin $\mathrm{V}$ and propidium iodide $(\mathrm{PI})$ staining of the thymocytes that were pretreated with pan-caspase inhibitor, Z-VAD and further incubated with PILR-Ig. As shown in Figure 4A, Z-VAD treatment significantly suppressed the apoptosis of thymocytes that were incubated with PILR-Ig, indicating that apoptosis through D4 ligation were dependent on caspase activity.

Next, to investigate the roles of caspase-3, caspase-7, and caspase-8 in D4-induced apoptosis, the activation of each caspase was tested by immunoblotting of the protein extract from PILR-lg treated thymocytes with the anti-caspase-3, anti-caspase-7, and anti-caspase-8 antibodies, respectively. The activated enzymes consisted of large and small subunits. Procaspase- 7 and 
A

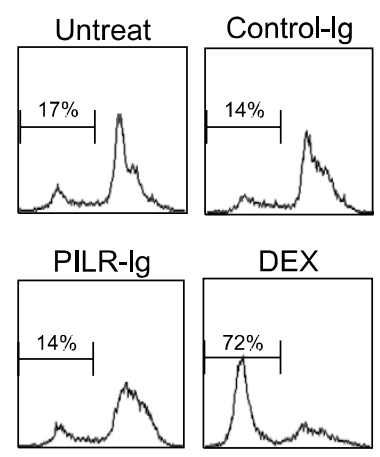

B

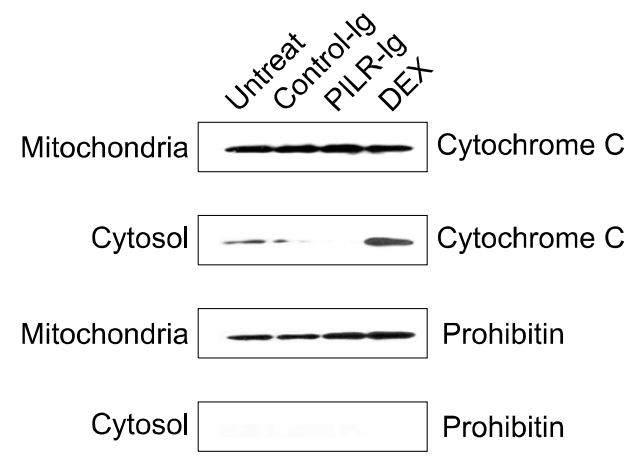

C

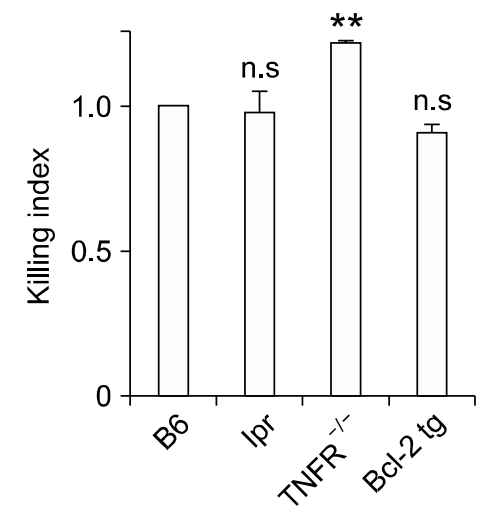

Figure 5. Apoptosis via D4 cross-linking is not mediated by mitochondrial events, known death receptors, or members of the Bcl-2 family. (A) Thymocytes treated with PILR-Ig or its isotype control, followed by treatment with secondary Ab, were stained with DiOC 6 , which reveals $\Delta \psi \mathrm{m}$. One representative flow cytometry result of two independent experiments is shown. (B) After treatment with PILR-Ig or the isotype control and secondary Ab, thymocytes were assessed for the presence of cytoplasmic cytochrome $\mathrm{C}$, as described in Methods. Membranes were sequentially reprobed for prohibitin (mitochondrial protein). Data are representative of two similar experiments. (C) Thymocytes were isolated from wild-type B6, Ipr/lpr (Fas-deficient), TNFR (p75)-deficient and Bcl-2-transgenic mice and cultured in the presence of PILR-Ig or isotype control, followed by treatment with secondary antibody for 6 $\mathrm{h}$, and the incidence of apoptosis was examined. To compare thymocyte apoptosis in different mouse strains with different internal controls, we normalized the individual responses to their respective controls. The normalized value is referred to as the killing index (see Methods). Data are expressed as the mean \pm SEM of triplicate samples pooled from three experiments. n.s. not significant, and ${ }^{* \star} P<0.01$ comparing control B6 group versus the other groups.

procaspase- 8 were cleaved to the active forms in the presence of D4 engagement, whereas procaspase-3 processing was not observed (Figures 4B-4D). However, caspase-7 was not activated when caspase-8 activity was blocked by IETD (caspase-8 inhibitor) (Figure 4E), which suggests that D4 ligation via PILR might lead to the sequential activation of caspase-8 and caspase-7.

\section{D4 cross-linking is not associated with mitochondrial membrane potential and is independent of Fas, TNFR, and Bcl-2}

The death signal is propagated by a caspase cascade that is initiated by the activation of large amounts of caspase-8, followed by the rapid cleavage of caspase- 3 and -7 , which in turn cleave vital substrates in the cells. In other cases, the caspase cascade cannot be propagated directly, but has to be amplified via the activation of mitochondria by caspase-8 (Li et al., 1998; Luo et al., 1998; Scaffidi et al., 1998). Therefore, we evaluated whether thymocyte apoptosis through D4 engagement is regulated by a mitochondrion-mediated signaling pathway via caspase-8 activation. Changes in the mitochondrial membrane potential $(\Delta \psi \mathrm{m})$ were measured by staining with DiOC6 (Zamzami et al., 1995a; Zamzami et al., 1995b). Unlike the dexamethasone-treated cells, which use the mitochondrion-dependent pathway, PILR-Ig-treated thymocytes exhibited no reduction in $\Delta \psi m$ (Figure 5A). To further confirm the relationship between D4 engagement and mitochondrial events, we tested whether D4 cross-linking resulted in the release of cytochrome $\mathrm{C}$, which is a key event in mitochondrial activation (Kroemer et al., 1995; Cai et al., 1998; Kuwana et al., 1998; Li et al., 1998; Luo et al., 1998). No cytosolic cytochrome $\mathrm{C}$ was detected in the PILR-Ig-treated thymocytes (Figure 5B), whereas the addition of dexamethasone triggered the release of cytochrome $\mathrm{C}$ into the cytosol (Marchetti et al., 2003). To rule out that the possibility that the appearance of cytochrome $\mathrm{C}$ in the cytosol was caused by leakage from fragmented or solubilized mitochondria, the intracellular localization of a mitochondrial protein, prohibitin, was examined and it was not in the supernatant fraction (Figure $5 B$ ). These data suggest that D4-induced apoptosis is entirely mitochondrion independent.

The ability of Fas and TNFR to mediate the apoptosis of DP thymocytes raised the possibility that TCR-independent, D4-induced apoptosis occurs through the engagement of Fas or TNFR (Punt et al., 1997). To address this possibility, we compared the abilities of engaged D4 to kill the thymocytes from wild B6, Fas-deficient Ipr, or TNFR p75 knockout mice. There was no substantial rescue in Fas or TNFR-deficient thymocytes from PILR-mediated apoptosis, which indicated that the Fas and TNFR pathways are not essential for D4-PILR-mediated cell death (Figure 5C). 
Bcl-2 family members play critical roles in many aspects of cell death. The overexpression of either $\mathrm{Bcl}-2$ or $\mathrm{Bcl}-\mathrm{X}_{\mathrm{L}}$ has been reported to protect DP thymocytes from apoptosis and lead to increased thymic cellularity (Bouillet et al., 1999). This report raised a possibility that the down-regulation of $\mathrm{Bcl}-2$ might be a candidate mechanism through which D4-induced apoptosis of DP thymocytes can be achieved. However, D4 engagement induced apoptosis in the thymocytes of mice that constitutively overexpress the anti-apoptotic $\mathrm{Bcl}-2$ protein in a degree comparable to that of the wild B6 control (Figure $5 \mathrm{C}$ ). This strongly suggests that the apoptotic signals generated by D4 engagement do not implicate Bcl-2-dependent cell death.

\section{Discussion}

Recently, murine D4 has been cloned and identified as the ligand of PILR, although the role of D4 in developing $T$ cells remains unknown (Shiratori et al., 2004). Regulated D4 expression in thymocytes and the predominant expression of PILR on thymic stromal cells as the ligand of D4 strongly suggest a specific role for D4 in thymocyte development. In support of this hypothesis, we demonstrated that the cross-linking of D4 with PILR-Ig induces the apoptosis of thymocytes in the absence of TCR signals. Under these conditions, thymocytes undergo the characteristic sequential apoptotic process: (1) phosphatidylserine exposure (annexin $\mathrm{V}^{+}$); (2) activation of caspases (caspase-8 and caspase-7); and (3) internucleosomal DNA fragmentation. DP thymocytes were more sensitive to D4-induced apoptotic pathway than were DN and SP thymocytes. Given that the selection of thymocytes occurs mainly at the DP stage, it was worth observing the dominant effects of D4 ligation in this particular cell subset.

It has been reported that a number of cell surface molecules, including CD5, CD28, CD38, and CD43, can cooperate with TCR to kill thymocytes (Lesage et al., 1997; Jung et al., 2004). However, a few molecules such as CD8, CD24, and CD45 on the surfaces of thymocytes induce the apoptosis of thymocytes in the absence of TCR ligation (Lesage et al., 1997; Grebe et al., 2004; Jung et al., 2004), raising the possibility that the death of thymocytes including death by neglect could be induced by interactions between membrane proteins in the absence of MHC-TCR interaction. DP thymocytes underwent cell death after CD24 or CD45 cross-linking by specific antibodies. Ligation of CD8 on DP thymocytes, either with antibodies to CD8 or with MHC class
I-coated microspheres, led to rapid apoptosis (Grebe et al., 2004). Above all, the induction of the apoptosis of thymocytes via CD8 ligation, independently of TCR, with a physiological ligand (MHC I-coated microspheres), rather than an antibody, deserves close attention. However, this previous study could not overcome the limitation that the experiments were conducted in a single-cell suspension, rather than in an anatomically intact organ system. Furthermore, there is no overt accumulation of thymocytes in $\beta 2 \mathrm{~m}^{-/}, \mathrm{CD}^{-/}$, or $\mathrm{MHC}^{-/}$mice, suggesting that CD8-mediated apoptosis is neither the normal physiological mechanism nor an explanation for thymocyte apoptosis, especially death by neglect.

We showed that D4 is a strong candidate molecule for the induction of thymocyte apoptosis in a TCR-independent manner. High sensitivity of DP thymocytes to PILR-induced apoptosis and increased cellularity from TCR $\alpha^{-1-}$ FTOC with D4-FC treatment were clearly observed. This raised the possibility that D4 signals may play some role in the induction of thymocytes apoptosis including death by neglect. Many reports have suggested that thymic corticosteroids play a specific role in this kind of elimination. Adrenalectomized mice displayed increased thymic cellularity and DP cells exhibited sensitivity to corticosteroids in the absence of TCR stimulation (Vacchio et al., 1999). Signals from the TCR could antagonize the apoptotic effect of glucocorticoids, leading to the hypothesis that the combined signals from steroid hormones and TCR might determine the fate of individual thymocytes (Ashwell et al., 2000). However, a contradictory opinion came from the results of an experiment showing that mice reconstituted with glucocorticoid receptor-negative fetal liver cells displayed normal thymic composition and cellularity, which indicated normal levels of death by neglect in the system (Brewer et al., 2002). The results implied that glucocorticoids would not play a role in this process or that alternative corticosteroid receptors exist to mediate thymocyte cell death. Considering the controversy over the role of corticosteroids in thymic apoptosis, it is likely that corticosteroids are not the only molecules leading to the death by neglect of DP thymocytes. Cytokine withdrawal has also been considered as a mechanism for death of thymocytes in early developmental stage. The cytokine IL-7 has been reported to up-regulate anti-apoptotic proteins and enhance the survival of thymocytes (von Freeden-Jeffry et al., 1997). IL-7R $\alpha$-deficient mice have a 20 -fold decrease in thymic size and a vastly constricted peripheral T-cell pool (von Freeden-Jeffry et al., 1995). In IL-7R $\alpha$-deficient mice, Bcl-2 overexpre- 
ssion restores the normal production and function of T cells (Akashi et al., 1997). Bcl-2 overexpression was observed to prevent the death of thymocytes bearing TCR that could not bind to MHC molecules in their environment (Linette et al., 1994; Strasser et al., 1994). Additionally, the proapoptotic Bcl-2 family proteins Bax and Bak double-deficient DP thymocytes were resistant to cytokine withdrawal and glucocorticoid-induced apoptosis. These data suggest that the predominant role of IL-7R signaling is to block the bcl-2-regulated apoptosis pathway and that the Bcl-2 family members play a critical role in the apoptosis of thymocytes induced by cytokine withdrawal or glucocorticoid treatment (Rathmell et al., 2002). However, this did not seem to be the case in D4-induced apoptosis, in that cellular apoptosis still occurred in bcl-2-overexpressing thymocytes. Therefore, we suggest that the signaling through D4 via cross-linking with PILR-Ig is a novel and as yet unidentified pathway related to thymic apoptosis, distinct from IL-7R and corticosteroid pathways, which are tightly linked to $\mathrm{Bcl}-2$.

The thymocytes from mice deficient in Fas or TNFRp75, which are known death receptors for DP thymocytes apoptosis, had the same characteristic death response to PILR-Ig treatment. Based on these results, we concluded that D4 signaling would be different from signals amplified by death receptors, which is consistent with the previous report that death receptors most likely play a minor role in T cell selection (Newton et al., 1998).

Regarding that thymocytes were induced to TCR-independent apoptosis both by D4 and human CD99 molecule, the function of D4 is likely to be very similar to that of human CD99. However, there are a few different aspects between apoptosis that were induced by D4 or human CD99 despite we still do not know what caused these differences. Unlike the D4-induced apoptosis, the apoptosis by human CD99 displayed early mitochondrial alteration but it did not involve detectable DNA fragmentation (Bernard et al., 1997). Moreover, the apoptosis by human CD99 proceeded more slowly; it took $18 \mathrm{~h}$ for completion (Bernard et al., 1997), while the induction of thymocyte death required only 6 hours for D4. These functional distinctions in inducing apoptosis might be resulted by differences between species of these molecules, but in fact, the experimental condition of inducing apoptosis was not exactly identical; PILR, a natural ligand of D4 was used for the stimulation of D4, but human CD99 was engaged by its monoclonal antibody.

Here, we provide several lines of evidence to support the notion that signaling mediated by the interaction between the membrane proteins D4 and PILR constitutes an active death signal, which removes thymocytes, predominantly at double positive stage. Most notably, blocking the D4-PILR interaction repeatedly increased thymic cellularity in FTOC using TCR $\alpha^{-/-}$mice as well as wild type mice. Therefore, it is possible that apoptotic signals through interaction between D4 and PILR may take some part in death by neglect by providing non-selectable thymocytes with persistent and ubiquitous apoptotic signals. However, some findings are against to this hypothesis. First, the death caused by D4 ligation was already evident by $6 \mathrm{~h}$. The lifespan of DP thymocytes, however, that ultimately undergoes death by neglect is thought to be approximately 3.5 days (Huesmann et al., 1991). Second, caspase- 8 was the initiating caspase for D4-induced apoptosis. However, it had been reported that thymocytes undergo normal development in the caspase-8 knock-out mice (Salmena et al., 2003). Finally, the interaction of D4 and PILR also induced the death of DN and SP thymocytes, although the death rate at these stages was much lesser compared with that of DP thymocytes. Therefore, to clarify the physiological function of D4 during thymocyte development, sophisticated analysis would be required using D4 deficient mice.

\section{Methods}

\section{Mice}

C57BL/6 and BALB/c mice were purchased from Daehan Biolink (Chungbuk, Korea). Fas-defective Ipr mice, TNFR p75 knockout mice, and TCR $\alpha$-chain-deficient mice in the C57BL/6 background were purchased from the Jackson Laboratory (Bar Harbor, ME). Bcl-2-transgenic mice were a gift from Dr. H.W. Lee (Yonsei University College of Medicine, Korea) (Jung et al., 2004). All mice were maintained under specific pathogen-free conditions at the animal facility of the Center for Animal Resource Development, Seoul National University College of Medicine, and were between 4 and 10 weeks of age when analyzed.

\section{Antibodies and reagents}

The cDNA fragments that correspond to the leader gene segments and the extracellular domains of PILR were amplified by PCR. The cDNA was inserted into the plasmid to generate a dodecameric Ig fusion protein (Arthos et al., 2002). The 293 cells were transiently transfected with the plasmids, and culture supernatants were collected using standard methods. These fusion proteins were designated PILR-Ig. For the production of soluble D4-Fc or CD86-Fc fusion protein, the extracellular domain of D4 or CD86 was fused to the Fc region of human IgG1 via integration into the pSecTag vector (Invitrogen, Carlsbad, CA). A hybri- 
doma cell line was generated by the fusion of splenocytes from Sprague-Dawley rats immunized with mouse D4 proteins and SP2/0-Ag14 myeloma cells (designated D4). Rabbit anti-caspase- 3 and anti-caspase- 8 antibodies were purchased from Santa Cruz Biotechnology (Santa Cruz, $\mathrm{CA})$, and rabbit anti-prohibitin antibody was purchased from Lab Vision (Westinghouse, CA). All other Abs and FITC-labeled annexin $\mathrm{V}$ were purchased from DiNonA (Seoul, Korea) or BD PharMingen (San Jose, CA). We purchased 3,3'-dihexyloxacarbocyanine iodide ( $\mathrm{DiOC}_{6}$ ), etoposide, dexamethasone, and SEB from Sigma-Aldrich (St. Louis, MO). Caspase inhibitors such as z-VAD-fmk and $z$-IETD-fmk were obtained from Calbiochem (San Diego, CA).

\section{Cell preparation and culture}

Cell suspensions of thymocytes from 4- to 10-wk-old mice were prepared by mincing thymic tissues through a fine mesh screen. Each thymocyte subset was prepared by magnetic cell sorting using MACS microbeads (Miltenyi Biotec, Bergisch Gladbach, Germany), as recommended by the manufacturer. Briefly, the CD $4^{+} \mathrm{CD} 8^{+} \mathrm{DP}$ subset was prepared by positive selection using anti-CD8 microbeads, and anti-CD4 microbeads were used to collect CD4 ${ }^{+} \mathrm{SP}$ thymocytes. The $\mathrm{CD}^{-} \mathrm{CD} 8^{-} \mathrm{DN}$ thymocytes were isolated by the elimination of $\mathrm{CD}^{+}$or $\mathrm{CD}^{+}$thymocytes using MACS. The purity of the collected subsets, as determined by flow cytometry using anti-CD4 and anti-CD8 Abs, ranged from $90 \%$ to $98 \%$. The purified thymocytes were cultured in DMEM (Life Technologies, Rockville, MD) that was supplemented with $10 \%$ fetal bovine serum and 0.05 $\mathrm{mM} \beta$-mercaptoethanol.

\section{Flow cytometry}

Fresh cell suspensions of thymocytes were resuspended in FACS buffer $(1 \times$ PBS, $0.1 \%$ bovine serum albumin, $0.1 \%$ sodium azide). After staining with fluorescence-conjugated antibodies for $30 \mathrm{~min}$ at $4^{\circ} \mathrm{C}$, the live cells, gated as the propidium iodide (PI; Sigma, St. Louis, MO)-negative population, were analyzed using FACSCalibur (BD Biosciences, San Jose, CA) equipped with the CellQuest Pro software (BD Biosciences, San Jose, CA).

\section{Detection of apoptosis}

Apoptosis was determined by DNA fragmentation assay and flow cytometry. DNA for fragmentation assay were prepared and fractionated by electrophoresis on $3 \%$ agarose by a modification of the method of Kim et al. (Kim et al., 2009). For the detection of early and late apoptosis by flow cytometry, annexin V-FITC and PI were used. The cells were washed three times with binding buffer $(0.1 \mathrm{M}$ HEPES/NaOH [pH 7.4], $1.4 \mathrm{M} \mathrm{NaCl}, 25 \mathrm{mM} \mathrm{CaCl}_{2}$ ) and incubated for $30 \mathrm{~min}$ at room temperature in the dark in binding buffer that contained FITC-conjugated annexin V and $20 \mu \mathrm{g} / \mathrm{ml} \mathrm{PI}$. Specific cell death (percentage cell death) was calculated as a normalized value according to the following formula: [percentage of live cells (unstimulated) percentage of live cells (stimulated)]/percentage of live cells (unstimulated). To compare thymocyte apoptosis in response to D4-mediated signals among experimental groups with different internal controls, individual responses were normalized and expressed as a killing index. The killing index was calculated as follows: (percentage of cell death induced by PILR-Ig under experimental conditions)/ (percentage of cell death induced by PILR-Ig under control conditions). A killing index of 1.0 means that the indicated condition did not affect PILR-Ig-induced apoptosis.

The mitochondrial membrane potential $\Delta \psi \mathrm{m}$ is created by the asymmetric distribution of protons across the inner mitochondrial membrane, giving rise to a chemical $(\mathrm{pH})$ and electrical gradient (Green and Reed, 1998). To evaluate $\Delta \psi \mathrm{m}$ by flow cytometry, the cationic lipophilic fluorochrome $\mathrm{DiOC}_{6}$ (1) was used. A total of $1 \times 10^{5}$ thymocytes was incubated with $5 \mathrm{nM} \mathrm{DiOC}_{6}$ for $15 \mathrm{~min}$ at $37^{\circ} \mathrm{C}$ and analyzed immediately using FACSCalibur.

\section{RT-PCR}

Thymic stromal cells were prepared as described previously (Jenkinson et al., 1982). In brief, isolated thymic rudiments were cultured for 5 days in medium with 1.35 $\mathrm{mM}$ dGuo, causing the selective depletion of hematopoietic cells while allowing the survival of stromal elements. Purified cell populations and a plasmid that encodes the PILR gene were used as the template. RNA was extracted from the cells using Triazole (Invitrogen, Paisley, UK), and RT-PCR was performed using reverse transcriptase (Koschem). The following primer pairs were used for amplification: for PILR, sense primer 5'-GAATTCATGGCTTTGTTGATCTCGCTTCCTGGAG-3' and antisense primer 5'-CGGCCGACAACCCAACTGTGGTTTGCAGATCCAG-3'; for Lck, sense primer 5'-CCAGTCAGGAGCTTGAATCC-3' and antisense primer 5'-GGATGCTGGTGGGAGAGA-3'; and for $\beta$-actin, sense primer 5'-GCTCCGGCATGTGCAA-3' and antisense primer 5'-AGGATCTTCATGAGGTAGT-3'.

\section{Western blotting}

To evaluate caspase activation, cell lysates were prepared by dissolving the cells in lysis buffer that contained $1 \%$ Triton X-100, $10 \mathrm{mM}$ Tris-Cl (pH 7.5), $150 \mathrm{mM} \mathrm{NaCl}, 2 \mathrm{mM}$ EGTA, $50 \mathrm{mM} \beta$-glycerophosphate, $10 \mathrm{mM} \mathrm{NaF}$, and $1 \mathrm{mM}$ PMSF. A $100-\mu \mathrm{g}$ aliquot of each lysate was loaded onto SDS-PAGE gel, separated by electrophoresis, and transferred onto a nitrocellulose transfer membrane (Schleicher \& Schuell, Keene, NH). The blotted membranes were incubated with primary $A b$, followed by peroxidase-conjugated secondary $\mathrm{Ab}$. The specific bands were visualized using the ECL kit (Amersham Pharmacia Biotech, Buckinghamshire, UK).

\section{Immuoprecipitation}

Thymocytes and 293 cells transfected with D4 were washed in PBS and lysed in ice-cold lysis buffer that contained $1 \%$ digitonin, $12.5 \mathrm{mM}$ HEPES, $50 \mathrm{mM} \mathrm{NaCl}, 5$ $\mathrm{mM} \mathrm{MgCl}$, and $1 \mathrm{mM} \mathrm{PMSF}$. Cell lysates were immunoprecipitated with protein $G$ that was pre-incubated with PILR-Ig and the control Ig-fusion protein, separated on SDS-PAGE gels, and detected using the anti-D4 Ab. 


\section{Cell fractionation}

Cells were washed in PBS and resuspended in hypo-osmolar lysis buffer that contained $10 \mathrm{mM}$ HEPES (pH 7.5), $10 \mathrm{mM} \mathrm{MgCl}, 42 \mathrm{mM} \mathrm{KCl}$, and $1 \mathrm{mM}$ PMSF. Cells were subjected to three cycles of freezing in liquid nitrogen and thawing at $37^{\circ} \mathrm{C}$. After centrifugation for 10 min at $10,000 \mathrm{~g}$, supernatants (cytosolic fraction) were collected and placed on ice. The pellet (mitochondrial fraction) was resuspended in hypo-osmolar lysis buffer that contained $0.1 \%$ NP-40.

\section{Fetal thymic organ culture}

The fetal thymi from C57BL/6, BALB/c, and $\mathrm{TCR}^{-/-}$mice were removed and cultured on GD15.5 with various dosages of D4-Fc or control-Fc protein $(100 \mu \mathrm{g} / \mathrm{ml}$ or 200 $\mu \mathrm{g} / \mathrm{ml}$ ) for 5 days. Briefly, each fetal thymic lobe was cultured on a polycarbonate filter (pore size $0.8 \mu \mathrm{m}$; Millipore, Medford, MA) in RPMI 1640 medium that was supplemented with $10 \%$ fetal bovine serum. After 5 days of culture, the lobes were harvested and single-cell suspensions were prepared for counting and analyzed for the expression of CD3, CD4, CD8, HSA, TCR $\gamma \delta$, and TCRV $\beta 3 / 8$ by flow cytometry using FACSCalibur.

Thymic lobes from BALB/c mice were cultured in the presence of D4-Fc or control-Fc for 7 days, and SEB (50 $\mu \mathrm{g} / \mathrm{ml}$ ) or PBS was added for the final $40 \mathrm{~h}$ of the culture period. As described above, thymocytes were collected, counted and analyzed for the expression of CD3, CD4, CD8, and TCRV $\beta 3 / 8$ by flow cytometry.

\section{Statistics}

Statistical significance was analyzed using the program Prism 4.0. Student's $t$-tests were used to determine the $P$-value when comparing two groups. $P$-values $<0.05$ were considered significant.

\section{Acknowledgments}

We thank Dr. James Arthos in NIH of USA for providing plasmid to generate a dodecameric Ig fusion protein. We thank Dr. Hanwoong Lee in Yonsei University for providing bcl-2 transgenic mice. This work was supported by the National Research Foundation (NRF) through the Tumor Immunity Medical Research Center (TIMRC) at Seoul National University College of Medicine (R13-2002-02501003-0).

\section{References}

Akashi $\mathrm{K}$, Kondo $\mathrm{M}$, von Freeden-Jeffry $\mathrm{U}$, Murray $\mathrm{R}$, Weissman IL. Bcl-2 rescues T lymphopoiesis in interleukin-7 receptor-deficient mice. Cell 1997;89:1033-41

Arthos J, Cicala C, Steenbeke TD, Chun TW, Dela Cruz C, Hanback DB, Khazanie P, Nam D, Schuck P, Selig SM, Van Ryk D, Chaikin MA Fauci AS. Biochemical and biological characterization of a dodecameric CD4-lg fusion protein: implications for therapeutic and vaccine strategies. J Biol

\section{Chem 2002;277:11456-64}

Ashkenazi A, Dixit VM. Death receptors: signaling and modulation. Science 1998;281:1305-8

Ashwell JD, Lu FW, Vacchio MS. Glucocorticoids in T cell development and function. Annu Rev Immunol 2000; 18:309-45

Bernard G, Zoccola D, Deckert M, Breittmayer JP, Aussel C, Bernard A. The E2 molecule (CD99) specifically triggers homotypic aggregation of CD4+ CD8+ thymocytes. J Immunol 1995;154:26-32

Bernard G, Breittmayer JP, de Matteis M, Trampont P, Hofman P, Senik A, Bernard A. Apoptosis of immature thymocytes mediated by E2/CD99. J Immunol 1997;158: 2543-50

Bouillet P, Metcalf D, Huang DC, Tarlinton DM, Kay TW, Kontgen F, Adams JM, Strasser A. Proapoptotic Bcl-2 relative Bim required for certain apoptotic responses, leukocyte homeostasis, and to preclude autoimmunity. Science 1999;286:1735-8

Brewer JA, Kanagawa O, Sleckman BP, Muglia LJ. Thymocyte apoptosis induced by $T$ cell activation is mediated by glucocorticoids in vivo. J Immunol 2002;169:1837-43

Cai J, Yang J, Jones DP. Mitochondrial control of apoptosis: the role of cytochrome c. Biochim Biophys Acta 1998; 1366:139-49

Chan SH, Cosgrove D, Waltzinger C, Benoist C, Mathis D. Another view of the selective model of thymocyte selection. Cell 1993;73:225-36

Choi EY, Park WS, Jung KC, Kim SH, Kim YY, Lee WJ, Park $\mathrm{SH}$. Engagement of CD99 induces up-regulation of TCR and MHC class I and II molecules on the surface of human thymocytes. J Immunol 1998;161:749-54

Cohen JJ. Programmed cell death in the immune system. Adv Immunol 1991;50:55-85

Cresswell P. Proteases, processing, and thymic selection. Science 1998;280:394-5

Crump AL, Grusby MJ, Glimcher LH, Cantor H. Thymocyte development in major histocompatibility complex-deficient mice: evidence for stochastic commitment to the CD4 and CD8 lineages. Proc Nati Acad Sci USA 1993;90:10739-43

Ellis NA, Ye TZ, Patton S, German J, Goodfellow PN, Weller P. Cloning of PBDX, an MIC2-related gene that spans the pseudoautosomal boundary on chromosome Xp. Nat Genet 1994;6:394-400

Frankel WN, Rudy C, Coffin JM, Huber BT. Linkage of Mls genes to endogenous mammary tumour viruses of inbred mice. Nature 1991;349:526-8

Grebe KM, Clarke RL, Potter TA. Ligation of CD8 leads to apoptosis of thymocytes that have not undergone positive selection. Proc Nati Acad Sci USA 2004;101:10410-5

Green DR, Reed JC. Mitochondria and apoptosis. Science 1998;281:1309-12

Hirata H, Takahashi A, Kobayashi S, Yonehara S, Sawai H, Okazaki T, Yamamoto K, Sasada M. Caspases are activated 
in a branched protease cascade and control distinct downstream processes in Fas-induced apoptosis. J Exp Med 1998;187:587-600

Huesmann M, Scott B, Kisielow P, von Boehmer H. Kinetics and efficacy of positive selection in the thymus of normal and T cell receptor transgenic mice. Cell 1991;66:533-40

Jenkinson EJ, Franchi LL, Kingston R, Owen JJ. Effect of deoxyguanosine on lymphopoiesis in the developing thymus rudiment in vitro: application in the production of chimeric thymus rudiments. Eur J Immunol 1982;12:583-7

Jenkinson EJ, Kingston R, Owen JJ. Newly generated thymocytes are not refractory to deletion when the alpha/beta component of the $T$ cell receptor is engaged by the superantigen staphylococcal enterotoxin B. Eur J Immunol 1990;20:2517-20

Jung KC, Park WS, Kim HJ, Choi EY, Kook MC, Lee HW, Bae Y. TCR-independent and caspase-independent apoptosis of murine thymocytes by CD24 cross-linking. J Immunol 2004;172:795-802

Kim K, Choi YH, Kim HH, Cheong J. The orphan nuclear receptor SHP inhibits apoptosis during the monocytic differentiation by inducing p21WAF1. Exp Mol Med 2009;41:429-39

Krammer PH. CD95's deadly mission in the immune system. Nature 2000;407:789-95

Kroemer G, Petit P, Zamzami N, Vayssiere JL, Mignotte B. The biochemistry of programmed cell death. FASEB $\mathrm{J}$ 1995;9:1277-87

Kuwana T, Smith JJ, Muzio M, Dixit V, Newmeyer DD, Kornbluth S. Apoptosis induction by caspase- 8 is amplified through the mitochondrial release of cytochrome c. J Biol Chem 1998;273:16589-94

Lesage S, Steff AM, Philippoussis F, Page M, Trop S, Mateo $\mathrm{V}$, Hugo P. CD4+ CD8+ thymocytes are preferentially induced to die following CD45 cross-linking, through a novel apoptotic pathway. J Immunol 1997;159:4762-71

$\mathrm{Li} \mathrm{H}$, Zhu H, Xu CJ, Yuan J. Cleavage of BID by caspase 8 mediates the mitochondrial damage in the Fas pathway of apoptosis. Cell 1998;94:491-501

Linette GP, Grusby MJ, Hedrick SM, Hansen TH, Glimcher $\mathrm{LH}$, Korsmeyer SJ. Bcl-2 is upregulated at the CD4+ CD8+ stage during positive selection and promotes thymocyte differentiation at several control points. Immunity 1994;1:197-205

Luo X, Budihardjo I, Zou H, Slaughter C, Wang X. Bid, a Bcl2 interacting protein, mediates cytochrome $\mathrm{c}$ release from mitochondria in response to activation of cell surface death receptors. Cell 1998;94:481-90

Marchetti MC, Di Marco B, Cifone G, Migliorati G, Riccardi C. Dexamethasone-induced apoptosis of thymocytes: role of glucocorticoid receptor-associated Src kinase and caspase-8 activation. Blood 2003;101:585-93

Matsuki Y, Zhang HG, Hsu HC, Yang PA, Zhou T, Dodd CH, Cecconi F, Gruss P, Tadakuma T, Mountz JD. Different role of Apaf-1 in positive selection, negative selection and death by neglect in foetal thymic organ culture. Scand $\mathrm{J}$ Immunol

\section{2;56:174-84}

Minter LM, Osborne BA. Cell death in the thymus--it's all a matter of contacts. Semin Immunol 2003;15:135-44

Newton K, Harris AW, Bath ML, Smith KG, Strasser A. A dominant interfering mutant of FADD/MORT1 enhances deletion of autoreactive thymocytes and inhibits proliferation of mature T lymphocytes. EMBO J 1998;17:706-18

Park SH, Shin YK, Suh YH, Park WS, Ban YL, Choi HS, Park HJ, Jung KC. Rapid divergency of rodent CD99 orthologs: implications for the evolution of the pseudoautosomal region. Gene 2005;353:177-88

Punt JA, Havran W, Abe R, Sarin A, Singer A. T cell receptor (TCR)-induced death of immature CD4+CD8+ thymocytes by two distinct mechanisms differing in their requirement for CD28 costimulation: implications for negative selection in the thymus. J Exp Med 1997;186:1911-22

Rathmell JC, Thompson CB. The central effectors of cell death in the immune system. Annu Rev Immunol 1999;17:781-828

Rathmell JC, Lindsten T, Zong WX, Cinalli RM, Thompson $\mathrm{CB}$. Deficiency in Bak and Bax perturbs thymic selection and lymphoid homeostasis. Nat Immunol 2002;3:932-9

Salmena L, Lemmers B, Hakem A, Matysiak-Zablocki E, Murakami K, Au PY, Berry DM, Tamblyn L, Shehabeldin A Migon E, Wakeham A, Bouchard D, Yeh WC, McGlade JC, Ohashi PS, Hakem R. Essential role for caspase 8 in T-cell homeostasis and T-cell-mediated immunity. Genes Dev 2003;17:883-95

Scaffidi C, Fulda S, Srinivasan A, Friesen C, Li F, Tomaselli KJ, Debatin KM, Krammer PH, Peter ME. Two CD95 (APO-1/Fas) signaling pathways. EMBO J 1998;17:1675-87

Schenkel AR, Mamdouh Z, Chen X, Liebman RM, Muller WA. CD99 plays a major role in the migration of monocytes through endothelial junctions. Nat Immunol 2002;3:143-50

Shiratori I, Ogasawara K, Saito T, Lanier LL, Arase H. Activation of natural killer cells and dendritic cells upon recognition of a novel CD99-like ligand by paired immunoglobulin-like type 2 receptor. J Exp Med 2004;199: 525-33

Strasser A, Harris AW, von Boehmer H, Cory S. Positive and negative selection of $\mathrm{T}$ cells in T-cell receptor transgenic mice expressing a bcl-2 transgene. Proc Nati Acad Sci USA 1994; $91: 1376-80$

Vacchio MS, Lee JY, Ashwell JD. Thymus-derived glucocorticoids set the thresholds for thymocyte selection by inhibiting TCR-mediated thymocyte activation. J Immunol 1999;163:1327-33

von Freeden-Jeffry U, Vieira P, Lucian LA, McNeil T, Burdach SE, Murray R. Lymphopenia in interleukin (IL)-7 gene-deleted mice identifies IL-7 as a nonredundant cytokine. J Exp Med 1995;181:1519-26

von Freeden-Jeffry U, Solvason N, Howard M, Murray R. The earliest T lineage-committed cells depend on IL-7 for Bcl-2 expression and normal cell cycle progression. Immunity 1997;7:147-54

Wallach D, Varfolomeev EE, Malinin NL, Goltsev YV, 
Kovalenko AV, Boldin MP. Tumor necrosis factor receptor and Fas signaling mechanisms. Annu Rev Immunol 1999;17:331-67

Zamzami N, Marchetti P, Castedo M, Zanin C, Vayssiere JL, Petit PX, Kroemer G. Reduction in mitochondrial potential constitutes an early irreversible step of programmed lymphocyte death in vivo. J Exp Med 1995a;181:1661-72

Zamzami N, Marchetti P, Castedo M, Decaudin D, Macho A, Hirsch T, Susin SA, Petit PX, Mignotte B, Kroemer G. Sequential reduction of mitochondrial transmembrane potential and generation of reactive oxygen species in early programmed cell death. J Exp Med 1995b;182:367-77 